LA-UR-01-5259

Approved for public release; distribution is unlimited.

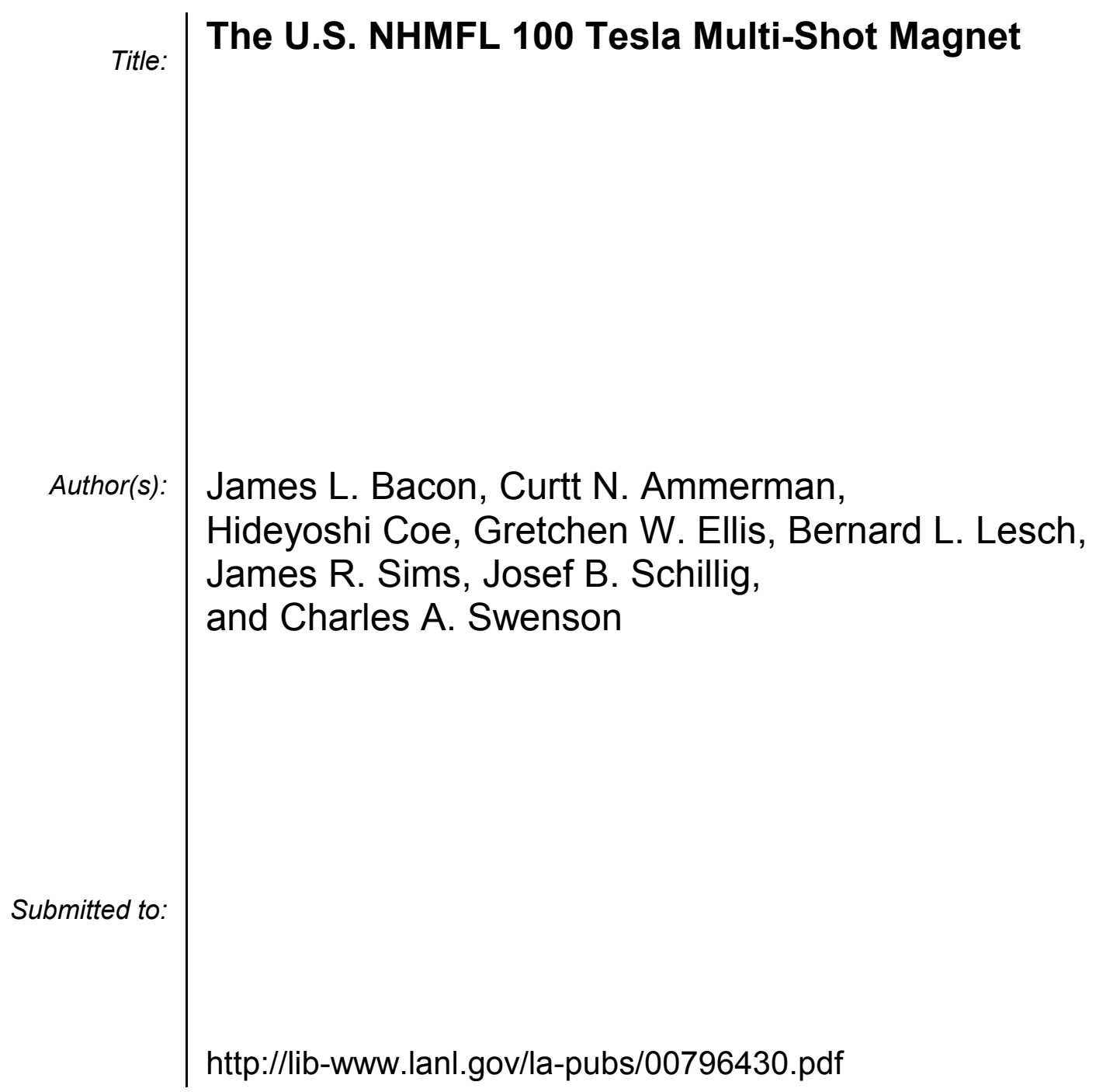

Los Alamos National Laboratory, an affirmative action/equal opportunity employer, is operated by the University of California for the U.S. Department of Energy under contract W-7405-ENG-36. By acceptance of this article, the publisher recognizes that the U.S. Government retains a nonexclusive, royaltyfree license to publish or reproduce the published form of this contribution, or to allow others to do so, for U.S. Government purposes. Los Alamos National Laboratory requests that the publisher identify this article as work performed under the auspices of the U.S. Department of Energy. Los Alamos National Laboratory strongly supports academic freedom and a researcher's right to publish; as an institution, however, the Laboratory does not endorse the viewpoint of a publication or guarantee its technical correctness. 


\title{
The U.S. NHMFL 100 Tesla Multi-Shot Magnet
}

\author{
James L. Bacon, Curtt N. Ammerman, Hideyoshi Coe, Gretchen W. Ellis, Bernard L. Lesch, \\ James R. Sims, Josef B. Schillig, Charles A. Swenson
}

\begin{abstract}
${ }^{1}$ Abstract-- The design, analysis and fabrication progress of the $100 \mathrm{~T}$ Multi-Shot Magnet is described. The description includes the structural analysis of the outer coil set, the fabrication of the $100 \mathrm{~T}$ prototype coil 1 , the fabrication of a coil 1 test shell, and the analysis of the electrical busbar assembly. Fabrication issues and their solutions are presented. This magnet will be installed as part of the user facility research equipment at the U.S. National High Magnetic Field Laboratory (NHMFL) Pulsed Field Facility at Los Alamos National Laboratory.
\end{abstract}

\section{Index Terms-- high field, pulsed magnet, 100 tesla}

\section{INTRODUCTION}

The $100 \mathrm{~T}$ multi-shot (100 T MS) magnet, now under fabrication, will provide a $100 \mathrm{~T}$ pulsed field in a $15 \mathrm{~mm}$ diameter bore. The magnet will consist of an outer coil set which will produce a platform field of $44.5 \mathrm{~T}$ and an insert coil, which will produce the remainder. Figure 1 is a graph of a simulated pulse. A converter-based power supply will power the outer coil set and a capacitor bank will drive the insert coil. The magnet is suspended in a cryogenic dewar (liquid nitrogen is used as the coolant). Details concerning the design and operation of the magnet can be found in [1] - [3].

\section{Magnet System}

\section{A. Experimental Bay}

Fig. 2 shows the future placement of the magnet in the pit. The pit is open to the rest of a large high bay but will be

Manuscript received September 24, 2001. This work was supported by US Department of Energy, Division of Materials Sciences and the US National Science Foundation through the NHMFL.

James L Bacon, Curtt N. Ammerman, Hideyoshi Coe, Gretchen W. Ellis, Josef B. Schillig, and James R. Sims are with the National High Magnetic Field Laboratory (NHMFL), Los Alamos National Laboratory, Los Alamos, NM 87545, USA (telephone: 505-665-4656, 505-665-0164, 505-667-6674, 505-665-6787, 505-665-1667, 505-665-1975, email: bacon@lanl.gov, ammerman@lanl.gov, coe@lanl.gov, gellis@lanl.gov, jschillig@lanl.gov, jsims@lanl.gov).

Bernard L. Lesch and Charles A. Swenson are with the National High Magnetic Field Laboratory (NHMFL), 1800 E. Paul Dirac Drive, Tallahassee, FL 32306, USA (telephone: 850-644-5109, 505-644-1623, email: lesch@magnet.fsu.edu, swenson@magnet.fsu.edu).

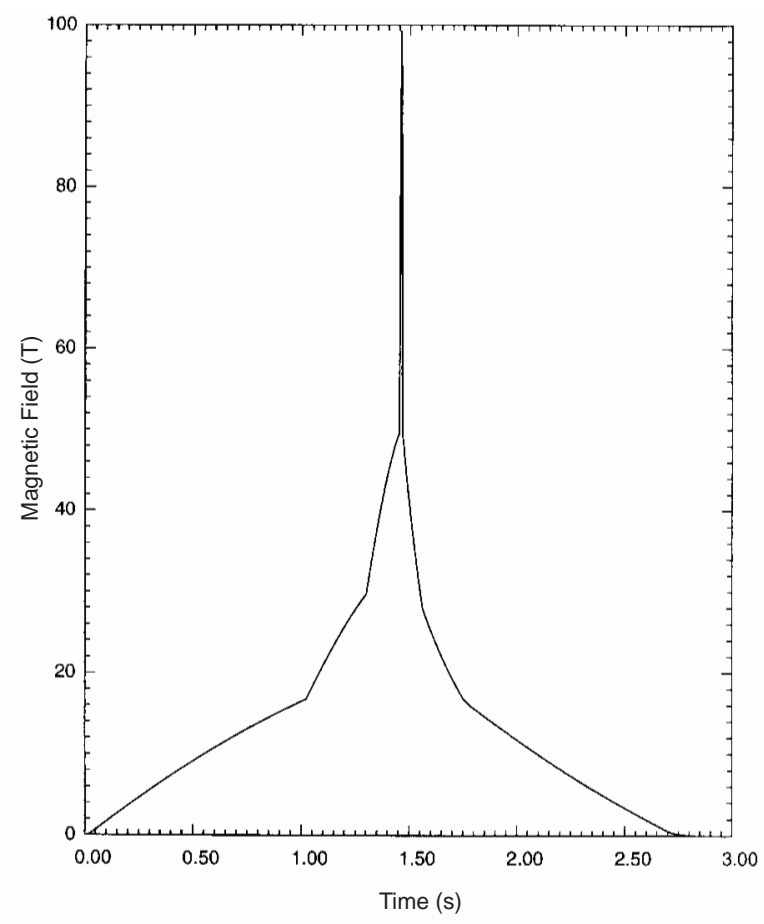

Fig. 1. Simulated $100 \mathrm{~T}$ pulse from $100 \mathrm{~T}$ MS magnet.

surrounded by a concrete blast wall with a stainless steel mesh ceiling to contain any fragments in the case of a high-energy magnet failure [4]. A two-tiered fiberglass platform provides access to the magnet lid and to the experiment cryostat. Electrical busbars, nitrogen piping, and instrument cables run

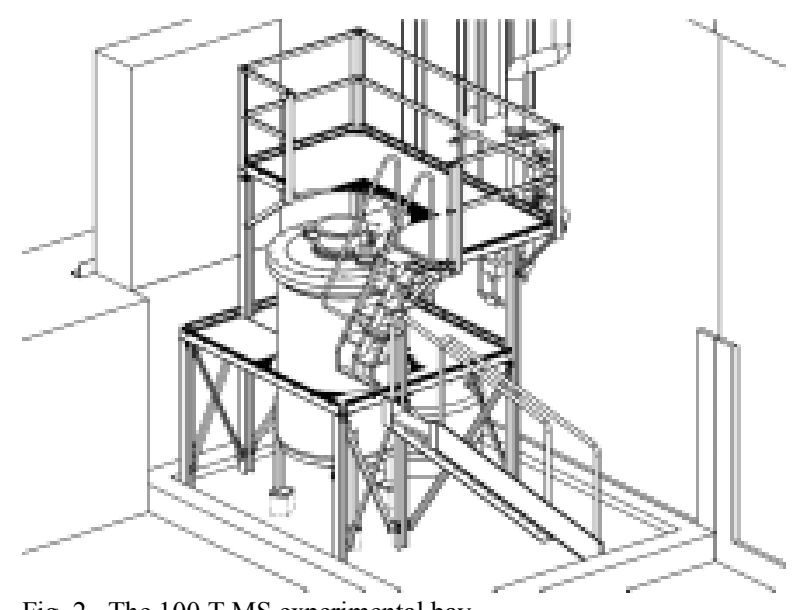

Fig. 2. The $100 \mathrm{~T}$ MS experimental bay. 
down the north wall of the pit (the south and east walls have been removed from this view).

\section{B. Outer Coil Set}

\section{1) Description}

The outer coil set consists of 7 coils divided into 3 separate electrical sections powered by a synchronous motor/generator through combinations of seven ac-de converters. A coil is comprised of a winding inserted into a steel shell and then vacuum impregnated with epoxy. The coils are numbered 1 through 7 starting at the innermost radial coil and progressing to the outermost radial coil. Section 1 consists of coils 1 and 2 connected in series, section 2 consists of coils 3 and 4 connected in series, and section 3 consists of coils 5, 6 and 7 connected in series. The sections are energized in stages; section 3 first, followed by section 2 and finally by section 1 .

The coils are nested and mounted in a NEMA G-10 epoxy fiberglass laminate and stainless steel frame. The frame is suspended inside a dewar which is cooled to $77 \mathrm{~K}$ operating temperature. The coils and their frame are free to expand and contract axially inside the dewar.

\section{2) Analysis and Testing}

A 1/4 magnetic axi-symmetric model of the outer coil set and surrounding space was created with Cosmos/ $\mathrm{M}^{\mathrm{TM}}$ commercial software. Current densities obtained from a circuit analysis code were applied to the conductor elements. The conductor forces and coil and shell temperatures obtained from the magnetic analysis were used in the structural analysis of the outer coil set. Two sets of forces and temperatures were applied to the model corresponding to an autofrettage pulse $(49.5 \mathrm{~T})$ followed by a nominal pulse $(47.00 \mathrm{~T})$. The autofrettage pulse is a singular event and is used to yield the conductors and drive them into a state of compression when unloaded (and thereby reducing their maximum tensile stress during a nominal pulse). The analysis was run in the non-linear mode to allow plastic deformation to occur during the autofrettage pulse and to simulate the Bauschinger effect during the nominal pulse.

The predicted stress ranges were used to guide the fatigue testing of the materials. One of the design goals for the outer coil set was to obtain a minimum of 10,000 full field pulses. The results of the fatigue testing performed at $77 \mathrm{~K}$ predict that all component materials can meet this design goal with the exception of the Glidcop AL-60 ${ }^{\mathrm{TM}}$ copper (UNS-C15760) used in coils 1 and 2 and the high yield 301 stainless steel (UNS-S30100 HY) used in the coils 1 and 2 shells.

A stress range of $660 \mathrm{MPa}$ tension to $440 \mathrm{MPa}$ compression was predicted for the conductor material in coils 1 and 2 (the most highly stressed coils) during a nominal pulse. Fatigue tests showed that samples failed between 3000 and 3500 cycles at this stress range. Results are conservative due to the introduction of bending stress (during compression) to the specimens during the testing. The use of either $\mathrm{CuNb}$ or $\mathrm{CuAg}$ could extend the life cycle but would present fabrication difficulties (leads cannot be silver brazed to the conductor).

Fatigue tests using the predicted stress range of $1895 \mathrm{MPa}$ tension to $366 \mathrm{MPa}$ tension were performed on the UNSS30100 HY. Fatigue life ranged from 9600 to 12500 cycles. The only other material known to have the necessary magnetic and thermal properties with a higher yield strength is MP35N (multiphase quaternary alloy: $35 \mathrm{Ni}-35 \mathrm{Co}-20 \mathrm{Cr}-$ $10 \mathrm{Mo}$ ) metal strip. There are no known sources of this material as wide as needed. The UNS-S30100 HY does not quite meet the design goal of 10,000 cycles but the life cycle of the coil 1 and 2 Glidcop AL-60 $0^{\mathrm{TM}}$ copper controls coil 1 and 2 replacement.

\section{3) Prototype Coil and Test Shell}

A prototype coil 1 was fabricated to demonstrate the manufacturability of the outer coil set and to serve as a learning tool. Figure 3 shows seven turns of insulated conductor wrapped under tension around a steel mandrel covered with release fabricate, two layers of Kapton ${ }^{\mathrm{TM}}$ sheet and three layers of E-glass fabric. The layer 1 lead can be seen making a right angle turn and penetrating the flange on the right side of the photograph. This lead was hand insulated with several layers of Kapton ${ }^{\mathrm{TM}}$ and E-glass ${ }^{\mathrm{TM}}$. The mandrel was wound with four layers, each having 58 turns, using G-10 filler pieces to support the layer-to-layer transitions. E-glass ${ }^{\mathrm{TM}}$ fabric is placed between each layer. The layer 4 lead pin was wound into the layer 1 socket, the conductor was cut and final layers of Kapton ${ }^{\mathrm{TM}}$ sheet, E-glass ${ }^{\mathrm{TM}}$ fabric and Armalon ${ }^{\mathrm{TM}}$ release fabric were applied.

Measurements of the diameter at the coaxial lead indicated a bulge of approximately $1.3 \mathrm{~mm}$ due to the difficulty of hand insulating the leads. This bulge presented problems during the insertion of the coil into the shell. The coaxial lead concept was discarded due to: 1) the difficulty in hand insulating, 2) the complexity of the leads, and 3) the undesirability of having a counterbore pocket in the bobbin (the coaxial leads extended past the outer surface of coils 1 - 4). An interlocking coil lead design based on the $60 \mathrm{~T}$ LP magnet [5] has been adopted.

Sixty-four layers of $0.393 \mathrm{~mm}$ thick UNS-S30100 HY sheet were wrapped over a Nitronic $40^{\mathrm{TM}}$ "bobbin". A wrap packing factor of $94 \%$ (the wrapped region was $94 \%$ metal and $6 \%$ air gap) was achieved. Prior to sheet wrapping, a $270^{\circ}$ section of 304 stainless steel, which tapered from a thickness of $0.39 \mathrm{~mm}$ to $0.13 \mathrm{~mm}$, was glued to the bobbin. The sheet was butted up to the thick end of the tapered 304 stainless steel and glued to the bobbin. The tapered steel section reduced the size of the discontinuity that would have occurred when the sheet is wrapped over itself from $0.39 \mathrm{~mm}$ to $0.13 \mathrm{~mm}$.

The steel sheet supply spool was mounted on a fixture, which allowed rotation and translation, so that supply spool to bobbin alignment was not critical. Steel guide rings were placed on the bobbin to guide the placement of the stainless steel sheet and establish straight edges. After the 64 layers of sheet metal were wrapped onto the bobbin the outer layer of sheet metal was spot welded onto the immediately underlying layer, the sheet metal path back to the supply spool was cut 
and the end tab of sheet metal on the bobbin was fillet welded to the underlying layer (see Fig. 4).

The structural models treated the overwrapped section of the shell as monolithic and simply derated the elastic modulus by $7 \%$ to reflect the existence of air gaps between layers. To validate the composite structure, a second shell was fabricated and will be tested using an internal pressure of $517 \mathrm{MPa}$ (maximum internal pressure on the shell during the autofrettage pulse), followed by loading until failure. A test fixture capable of providing this pressure loading on the shell at an operating temperature of $77 \mathrm{~K}$ is shown in Fig. 5. Pressure is applied to the shell by heating an aluminum thrust ring, which presses down on the top wedge and forces a set of shoes out into the shell. The follower is then rotated down until contact with the upper wedge block is made. The aluminum thrust ring is allowed to cool and contract, then the lock ring is tightened until contact with the aluminum thrust ring is established. The process is then repeated. Using a wedge angle of $10^{\circ}$, a maximum axial force of $237 \mathrm{MN}$ is required and a total travel of $0.028 \mathrm{~m}$. Sixteen cycles are required for a $0.305 \mathrm{~m}$ long aluminum thrust ring to produce $0.028 \mathrm{~m}$ of thermal growth when cycled between $80 \mathrm{~K}$ and $373 \mathrm{~K}$.

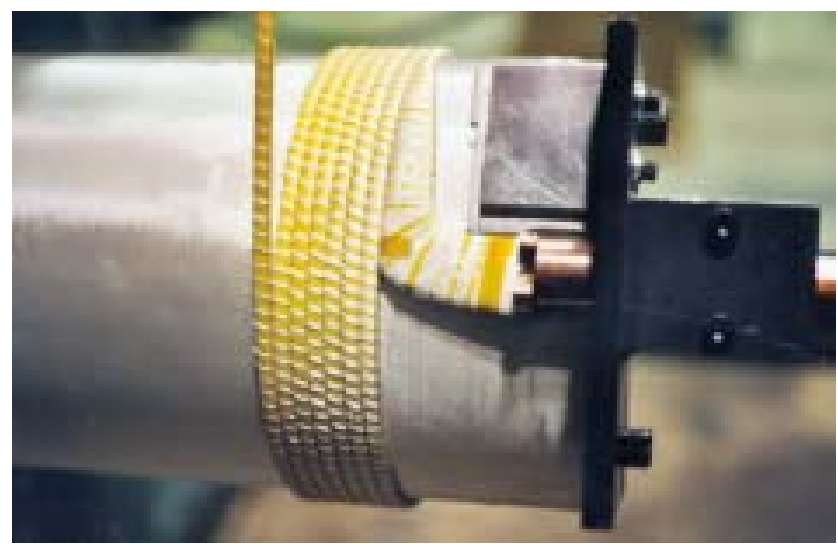

Fig. 3. The first seven layers of conductor wound onto the mandrel.

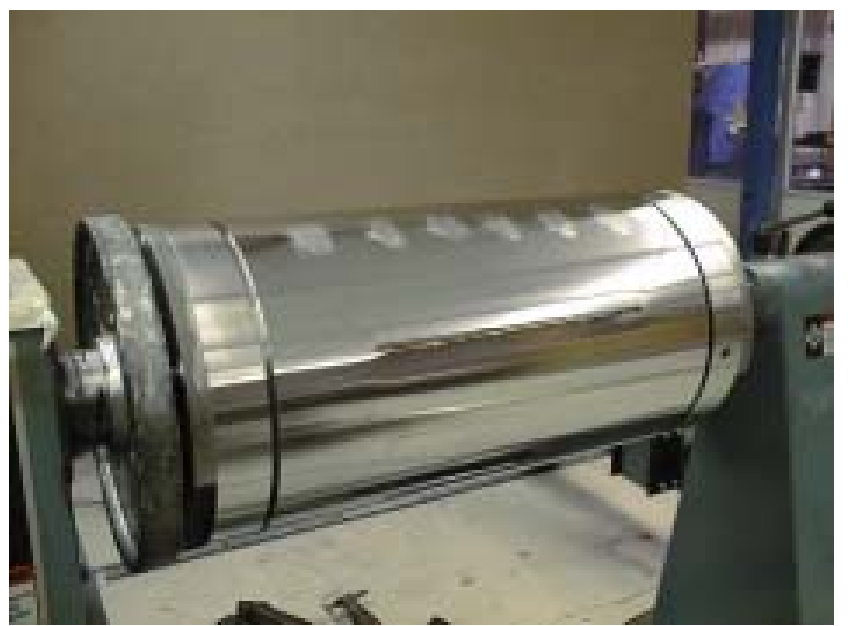

Fig. 4. The prototype bobbin wrapped with the 301 high yield stainless steel after welding.

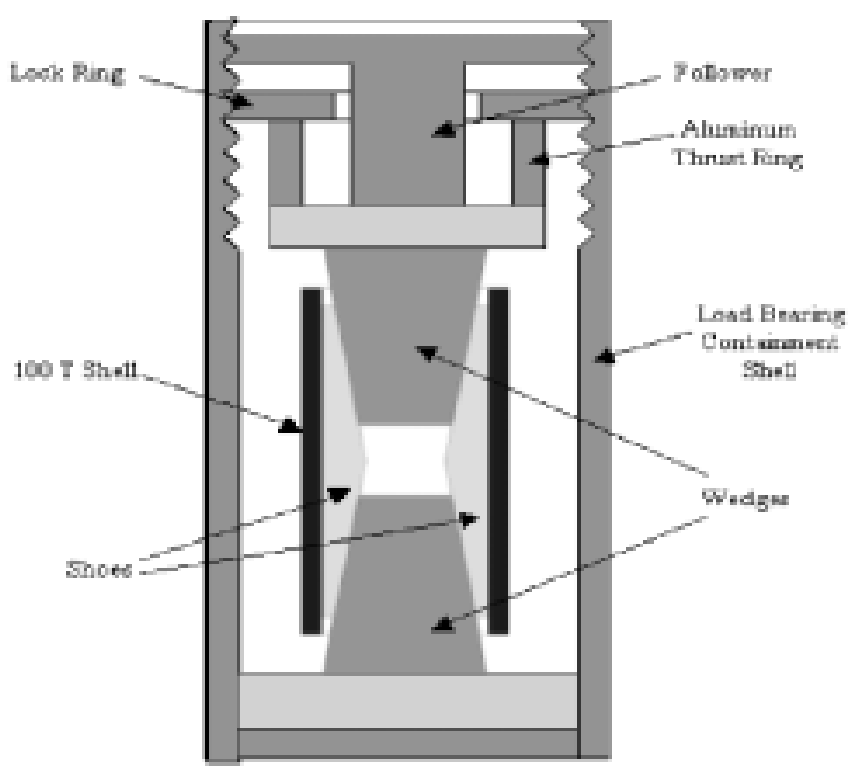

Fig. 5. A test fixture concept for applying the maximum operating pressure to a $100 \mathrm{~T}$ MS shell

\section{Insert Coil}

Four insert coil designs ranging from conservative to optimistic have been analyzed. All four concepts: 1) are 6 layer coils, 2) require $15.2 \mathrm{mF}$ bank with a drive voltage of $13.5 \mathrm{kV}, 3$ ) have $\mathrm{CuNb}$ conductors, and 4) are reinforced internally and externally with Zylon and MP35N. The first three concepts have $15 \mathrm{~mm}$ bores while the fourth has a 12 $\mathrm{mm}$ bore. The most conservative design would allow the magnet to produce $94.6 \mathrm{~T}$ while the most aggressive and the $12 \mathrm{~mm}$ bore design would allow the magnet to produce 100 T. Reference [3] contains greater detail.

\section{Dewar}

The outer coil set assembly will be supported by and contained in a liquid nitrogen (LN2) dewar as shown in Fig. 6. The dewar will be initially filled with 416 liters of LN2. Before the magnet is pulsed, the LN2 will be drained from the dewar to the catch tank. After the magnet is pulsed, the dewar is refilled with the LN2 in the catch tank, and lost LN2 will be added through a fill line in the dewar lid. Approximately 1 hour is required to cool the magnet back to the operating temperature after a full field pulse.

The $9072 \mathrm{~kg}$ magnet will be suspended in the dewar within a G-10 fiberglass frame structure. The frame structure is bolted to a structural support spool, which is suspended kinematically from the top flange of the dewar. The magnet is difficult to position due to its weight and size. Accurate positioning will be necessary to ensure concentricity of the outer coil set and the insert.

The magnet will be positioned through kinematic and jacking features on the magnet support spool. Three pins in the flange of the support spool will be engaged in 3 grooves in the dewar flange, allowing the magnet and dewar to remain coaxial while undergoing differential contraction. 


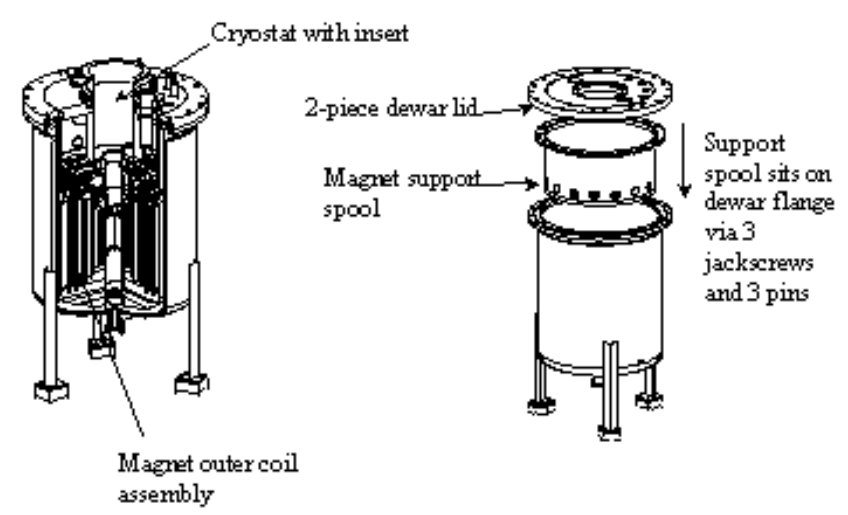

Fig. 6. The magnet is suspended inside the dewar from the magnet support spool.

Jacking screws will be used to support the magnet and adjust its angular orientation. These screws will sit in counterbores machined into the dewar flange.

The dewar was designed for two additional loads. First, the dewar must react forces of attraction between the magnet and the north wall of the magnet pit caused by the interaction of the magnet fringe field and steel rebar embedded in the wall. These forces were conservatively calculated to be on the order of $45 \mathrm{kN}$. Second, the dewar must be able to contain a pressure "pulse" of expanding Nitrogen gas on the order of $690 \mathrm{kPa}$. This fault scenario results from assuming a rapid deposition of energy into the $\mathrm{N} 2$ gas due to an arc.

Fabrication of the dewar, support spool, and liquid nitrogen catch tank were completed in June 2001.

\section{E. Busbar}

A nominal current of $20 \mathrm{kA}$ will be supplied to the $100 \mathrm{~T}$ MS magnet outer coil set via three electrical circuits. Current will be supplied to each coil group in a coaxial configuration, although this configuration must be interrupted at the transition between coils (see Fig. 7). The sections of busbar at the transition between coils will experience large $\mathrm{j} \mathrm{X} \mathrm{B}$ forces. Extensive structural analysis was performed for each coil group busbar. After several design and analysis iterations, predicted stresses were reduced to acceptable levels through

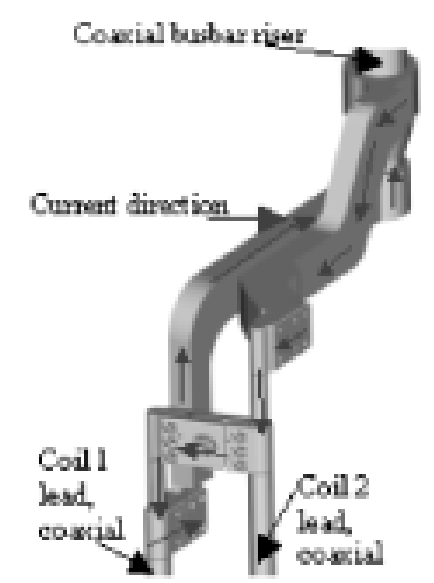

Fig. 7. The non-coaxial leads connecting coils 1 and 2 experience very large forces during a $100 \mathrm{~T}$ pulse

the use of a G-10 restraint system.

Additionally, the busbar was designed to accommodate the angular and positional misalignments inherent in the leads of each coil. The components were designed with oversized bolt holes and spherically articulating features that allow busbar assembly without custom machining and fitting of surfaces and bolt hole patterns. A busbar test stand has been fabricated to demonstrate that the components exhibit a sufficiently low contact resistance to carry the design current.

\section{SUMMARY}

The development of the $100 \mathrm{~T}$ MS has progressed significantly. Analysis of the outer coil set has been completed. The design of the outer coil set is nearing completion. Fabrication of a prototype coil 1 (inner most coil of the seven coils comprising the outer coil set) is in progress. The magnet dewar, nitrogen catch tank and magnet support spool have been fabricated, and a below grade concrete lined magnet pit has been constructed. Finally, a preliminary analysis of the insert coil has been completed.

\section{REFERENCES}

[1] J. R. Sims, et al., "First 100 T Non-Destructive Magnet," IEEE Transactions of Applied Superconductivity, vol. 10, pp. 510-513, March 2000.

[2] J. L. Bacon, et al., "First 100 T Non-Destructive Magnet Outer Coil Set," IEEE Transactions on Applied Superconductivity, vol. 10, pp. 514-517, March 2000.

[3] C. H. Swenson, B. L. Lesch, J. R. Sims, H. J. Schneider-Muntau, "Generation of pulsed magnetic fields: limitations, achievements, and prospects," IEEE Transactions on Applied Superconductivity, this issue.

[4] J. R. Sims, et al, "The U.S. NHMFL 60 T Long Pulse Magnet Failure", IEEE Transactions on Applied Superconductivity, this issue.

[5] J. R. Sims, et al, "Completion of the US NHMFL $60 \mathrm{~T}$ QuasiContinuous Magnet," Proceedings of the 15th Intl. Conf. on Magnet Technology, Beijing, China, October 20-24, 1997, pp. 635-641. 